\title{
Impaired basolateral sorting of pro-EGF causes isolated recessive renal hypomagnesemia
}

\author{
Wouter M. Tiel Groenestege,, ${ }^{1}$ Stéphanie Thébault,, Jenny van der Wijst, ${ }^{1}$ Dennis van den Berg, ${ }^{1}$ \\ Rob Janssen, ${ }^{1}$ Sabine Tejpar, ${ }^{2}$ Lambertus P. van den Heuvel, ${ }^{3}$ Eric van Cutsem, ${ }^{2}$ \\ Joost G. Hoenderop, ${ }^{1}$ Nine V. Knoers, ${ }^{4}$ and René J. Bindels ${ }^{1}$

\begin{abstract}
1Department of Physiology, Nijmegen Centre for Molecular Life Sciences, Radboud University Nijmegen Medical Centre, Nijmegen, The Netherlands. 2Digestive Oncology Unit, Department of Internal Medicine, University Hospital Gasthuisberg and Catholic University Leuven, Leuven, Belgium. ${ }^{3}$ Department of Pediatrics and ${ }^{4}$ Department of Human Genetics, Nijmegen Centre for Molecular Life Sciences, Radboud University Nijmegen Medical Centre, Nijmegen, The Netherlands.
\end{abstract}

\begin{abstract}
Primary hypomagnesemia constitutes a rare heterogeneous group of disorders characterized by renal or intestinal magnesium $\left(\mathrm{Mg}^{2+}\right)$ wasting resulting in generally shared symptoms of $\mathrm{Mg}^{2+}$ depletion, such as tetany and generalized convulsions, and often including associated disturbances in calcium excretion. However, most of the genes involved in the physiology of $\mathrm{Mg}^{2+}$ handling are unknown. Through the discovery of a mutation in the EGF gene in isolated autosomal recessive renal hypomagnesemia, we have, for what we believe is the first time, identified a magnesiotropic hormone crucial for total body $\mathrm{Mg}^{2+}$ balance. The mutation leads to impaired basolateral sorting of pro-EGF. As a consequence, the renal EGFR is inadequately stimulated, resulting in insufficient activation of the epithelial $\mathrm{Mg}^{2+}$ channel TRPM6 (transient receptor potential cation channel, subfamily $\mathrm{M}$, member 6) and thereby $\mathrm{Mg}^{2+}$ loss. Furthermore, we show that colorectal cancer patients treated with cetuximab, an antagonist of the EGFR, develop hypomagnesemia, emphasizing the significance of EGF in maintaining $\mathrm{Mg}^{2+}$ balance.
\end{abstract}

\section{Introduction}

Magnesium $\left(\mathrm{Mg}^{2+}\right)$ is established as a central electrolyte in a large number of cellular metabolic reactions, including DNA synthesis, neurotransmission, and hormone receptor binding. It is a component of GTPase and a cofactor for Na,K-ATPase, adenylate cyclase, phosphoinositide kinases, and phosphofructokinase (1). $\mathrm{Mg}^{2+}$ is also important for the regulation of parathyroid hormone release $(2,3)$. Accordingly, $\mathrm{Mg}^{2+}$ deficiency (plasma $\mathrm{Mg}^{2+}$ concentrations below $0.70 \mathrm{mM}$ ) has an effect on multiple body functions. Symptoms of $\mathrm{Mg}^{2+}$ deficiency are mostly related to muscle dysfunctioning, such as tetany, prolonged QT interval, and cardiac arrhythmias (4). Children with hypomagnesemia often present with tetany and/or convulsions. Hypomagnesemia is a problem frequently observed in more than $10 \%$ of hospitalized patients and occurrences can be as high as $65 \%$ in intensive care patients (5). A long-term complication seen in many adult patients with chronic hypomagnesemia is chondrocalcinosis, which can lead to impairment of joint function (4). $\mathrm{Mg}^{2+}$ deficiency can be secondary to systemic diseases (for instance, diabetes mellitus and Crohn disease) or to the use of osmotic agents, diuretics, and drugs such as cyclosporin and cisplatin (6). In addition, primary $\mathrm{Mg}^{2+}$ deficiency is observed in several monogenetic disorders. Failure of early diagnosis or noncompliance with treatment can be fatal or result in permanent neurological damage.

Nonstandard abbreviations used: DCT, distal convoluted tubule; FE, fractional excretion; IRH, isolated recessive renal hypomagnesemia; MDCK, Madin-Darby canine kidney; NCC, thiazide-sensitive sodium chloride cotransporter; pSAD, plasmid SAD; TRPM6, transient receptor potential cation channel, subfamily M, member 6 . Conflict of interest: The authors have declared that no conflict of interest exists. Citation for this article: J. Clin. Invest. 117:2260-2267 (2007). doi:10.1172/JCI31680.
The plasma $\mathrm{Mg}^{2+}$ concentration is regulated within a narrow range by changes in urinary $\mathrm{Mg}^{2+}$ excretion in response to altered uptake by the intestine. Thus, the kidney plays a key role in $\mathrm{Mg}^{2+}$ homeostasis $(4,7)$. Most renal reabsorption of $\mathrm{Mg}^{2+}$ occurs in the proximal tubule and the thick ascending limb of the loop of Henle via a passive paracellular transport process, but the fine-tuning of the $\mathrm{Mg}^{2+}$ excretion takes place in the distal convoluted tubule (DCT), where $\mathrm{Mg}^{2+}$ is reabsorbed via an active transcellular transport process $(6,7)$. Apical entry into DCT cells is mediated by the $\mathrm{Mg}^{2+}$-permeable channel TRPM6 (transient receptor potential cation channel, subfamily $\mathrm{M}$, member 6 ) driven by a favorable transmembrane voltage (8). The mechanism of basolateral $\mathrm{Mg}^{2+}$ transport into the interstitium is unknown. $\mathrm{Mg}^{2+}$ has to be extruded against an unfavorable electrochemical gradient, most likely by a $\mathrm{Na}^{+} / \mathrm{Mg}^{2+}$-dependent exchange mechanism and/or a $\mathrm{Mg}^{2+}$ ATPase. Finally, $3 \%-5 \%$ of the filtered $\mathrm{Mg}^{2+}$ is excreted in the urine.

Despite the critical role in $\mathrm{Mg}^{2+}$ handling, the exact mechanisms of transepithelial $\mathrm{Mg}^{2+}$ transport remain obscure. Studies of disorders with primary hypomagnesemia are very important to gaining more insight into the molecular and cellular mechanisms that underlie $\mathrm{Mg}^{2+}$ (re)absorption. Genetic studies in families with hereditary renal $\mathrm{Mg}^{2+}$ wasting syndromes have identified several genes that are either directly or indirectly involved in active $\mathrm{Mg}^{2+}$ handling. In the past few years, genetic studies of inborn errors of the $\mathrm{Mg}^{2+}$ balance revealed several new proteins unexpectedly involved in transepithelial $\mathrm{Mg}^{2+}$ transport in the DCT, e.g., thiazide-sensitive sodium chloride cotransporter (NCC), the $\gamma$ subunit of the $\mathrm{Na}, \mathrm{K}$-ATPase, and the previously mentioned epithelial $\mathrm{Mg}^{2+}$ channel, TRPM6 (9-12).

The aim of the present study was, therefore, to elucidate the gene defect and molecular mechanism underlying isolated reces- 
A

A । $\varnothing_{-}^{1} \square^{2}$

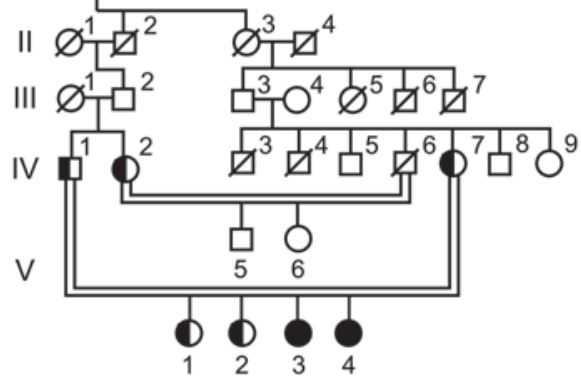

B

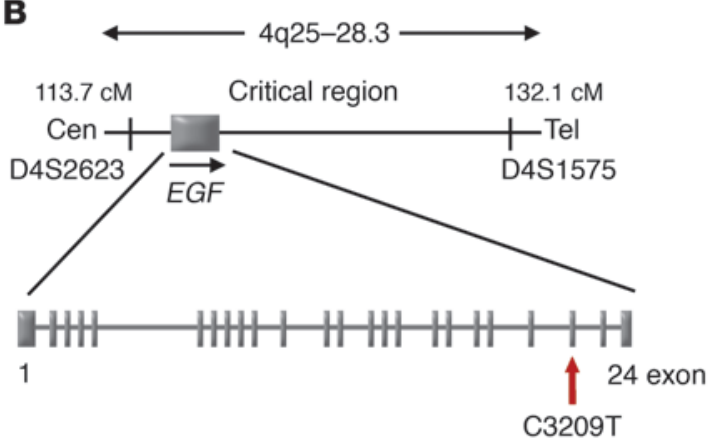

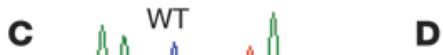

D

Extracellular

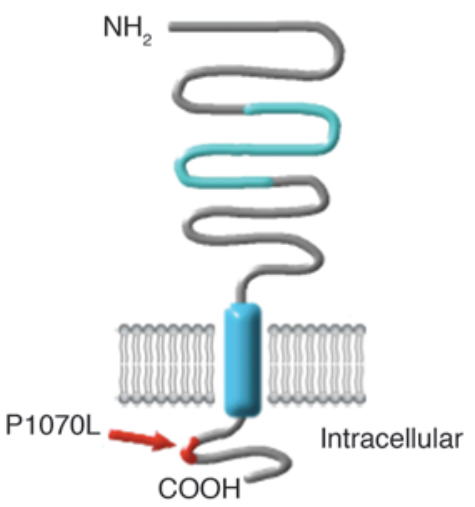

A G A A T C T T T A

E Human LWGAHYYRTQKLLSKNPKN PYEESSRDVRSRRPADTEDGM Chimpanzee LWGAHYYRTQKLLSKNPKN PYEESSRDVRSRRPADTEDGM Pig LWGAHYYRTQKLLSKNPKN PYEESGRDVSGIRPADGEAGM Cat LWGAHCYRTKKLPSKNLKN PYEESSRE--VSRPTDSEAGM Dog LGGAHCYRTKKLSSKNLKN PYEEPSREGSSSRPSDSEARM COW ----YYYRTQKWLLKNPKN PCEEPSRDVIRSRPADGEAGBlack rat MWGTYYYRTRKQLSESSKKPSEESSSNVSSNGPDSSGAGV Brown rat MWGTYYYRTRKQLSESSKK PSESSSNVSSNGPDSSGAGV Mouse P1070L

\section{Figure 1}

Molecular analysis of EGF. (A) Pedigree of the family in which specific members suffer from IRH. Filled symbols represent affected individuals, half-filled symbols are heterozygous for the mutation C3209T, and open symbols represent clinically unaffected family members not screened for the mutation. Slash marks indicate deceased individuals, and double lines show consanguinity. (B) Schematic representation of the critical interval between polymorphic markers D4S2623 and D4S1575 on chromosome 4q and the intron-exon structure of the EGF gene, depicting the identified mutation. The position of the polymorphic markers is indicated by vertical bars. The horizontal arrow below the schematical boxed representation of EGF depicted between the polymorphic markers indicates the localization and orientation of the EGF gene. Cen, centromeric; tel, telomeric. (C) Genomic mutation sequence analysis of EGF in wild-type, heterozygous, and affected individuals. The mutated nucleotide and resulting amino-acid change is shown under the affected individual's sequence. The black bar under that sequence indicates the mutated codon. The black bars under the sequences of the WT and heterozygous specify codon 1070 of EGF. The affected individuals both have a homozygous mutation C3209T in exon 22, resulting in the amino acid substitution P1070L. (D) Schematic model of pro-EGF, which resides as a type I membrane protein at the plasma membrane. The position of the P1070L mutation is depicted. (E) Sequence homology analysis of juxtamembrane domain and flanking residues. P1070 is strongly conserved among different species and forms the second proline of a basolateral PXXP sorting motif. The indicated colors represent functional conservation of the amino acids: red, small plus hydrophobic; blue, acidic; magenta, basic; green, hydroxyl plus amine plus basic; gray, others. TM, transmembrane domain.

sive renal hypomagnesemia (IRH), which is characterized by renal $\mathrm{Mg}^{2+}$ loss. To this end, a homozygosity-based mapping strategy and mutation detection was performed. In addition, the molecular mechanism of IRH was studied in vitro using patch clamp analysis and in vivo using clinical studies in humans.

\section{Results and Discussion}

IRH is characterized by low serum $\mathrm{Mg}^{2+}$ levels and mental retardation. Two affected sisters, V3 and V4 (Figure 1A), displayed low serum $\mathrm{Mg}^{2+}$ levels $(0.53-0.66 \mathrm{mM})$ in combination with urinary fractional excretion (FE) values of $\mathrm{Mg}^{2+}$ of $4.8 \%$ and $3.6 \%$, respectively. These values are well above an FE of $2 \%$, indicating renal $\mathrm{Mg}^{2+}$ wasting as previously described $(5,13)$. Thus, the fact that the urinary excretion of $\mathrm{Mg}^{2+}$ was in the normal range while serum $\mathrm{Mg}^{2+}$ values were hypomagnesemic points to a diminished tubular
$\mathrm{Mg}^{2+}$ reabsorption. Apart from hypomagnesemia, other biochemical abnormalities were not identified; in particular, there was no disturbance in urinary $\mathrm{Ca}^{2+}$ excretion (14). Both sisters showed psychomotor retardation during childhood, and presently they are moderately mentally retarded women who suffer from epileptic seizures. The previously identified genes involved in renal $\mathrm{Mg}^{2+}$ handling encoding the thiazide-sensitive sodium chloride cotransporter NCC (SLC12A3), paracellin-1 (CLDN16), the $\gamma$ subunit of the NaK-ATPase (FXYD2), and the epithelial $\mathrm{Mg}^{2+}$ channel (TRPM6) have been excluded for these 2 affected sisters (Figure 1A) $(8,11$, $12,15)$. To determine the disease locus of this consanguineous family, a homozygosity-based mapping strategy with a set of highly polymorphic microsatellite markers spread over the genome was performed and followed by fine mapping. This resulted in the identification of a critical linkage interval with LOD score 2.66 


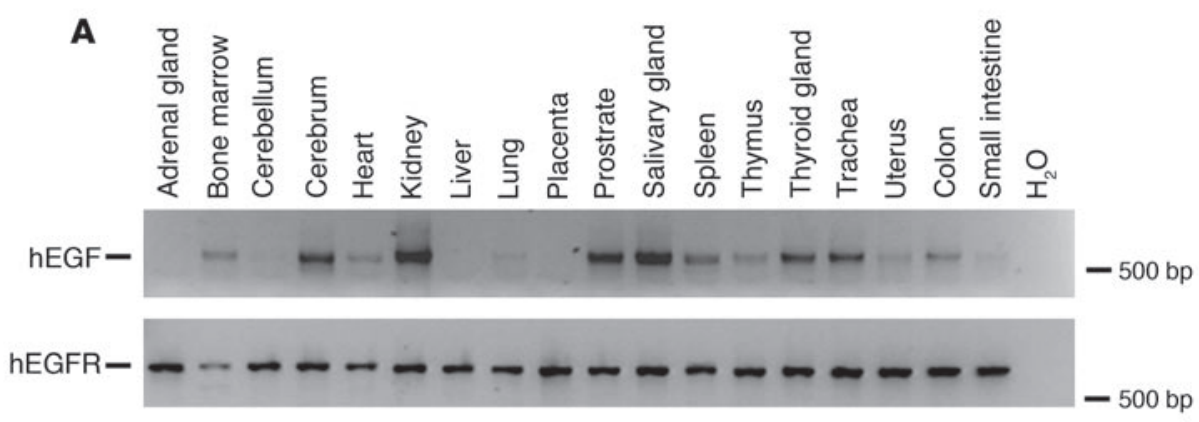

B
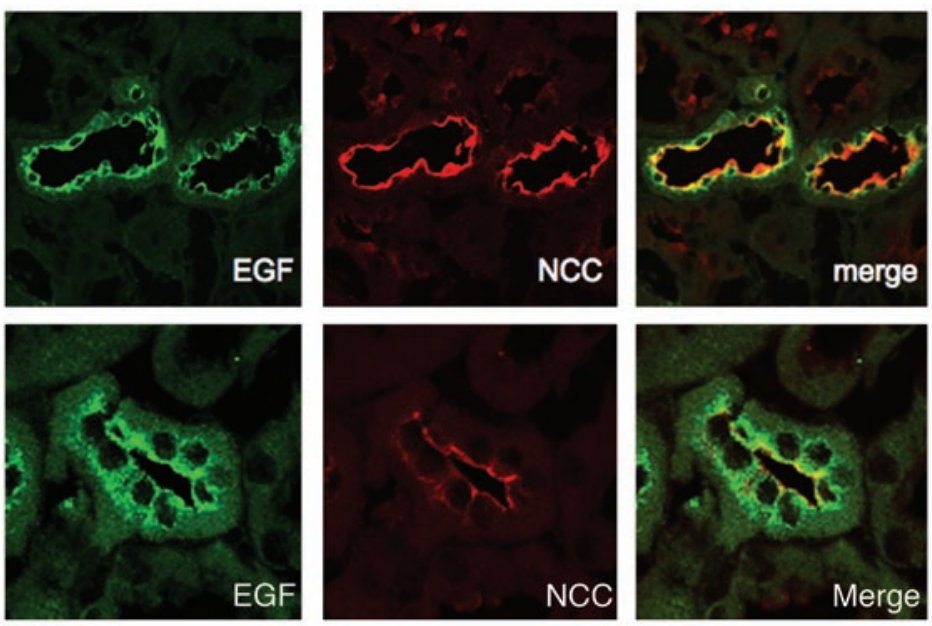

\section{Figure 2}

Expression profiling of EGF and EGFR. (A) The expression of EGF and EGFR mRNA was determined by RT-PCR on various human tissues. Signals for EGF were detected in, e.g., kidney, salivary gland, prostate, and cerebrum whereas no signals were detected in the adrenal gland, cerebellum, liver, lung, and placenta. The EGFR showed a ubiquitous expression pattern since PCR amplification products were obtained in all tissues tested. hEGF, human EGF; hEGFR, human EGFR. (B) Immunohistochemical analysis of EGF (green) and thiazidesensitive sodium chloride cotransporter (NCC, red) in rat kidney sections (upper panel, overview of a cortical region; lower panel, magnified image of an immunopositive tubule). EGF colocalized with NCC, a marker for the DCT. Original magnification, $\times 180$ (B, top panels); ×360 (B, bottom panels).
$(P<0.004)$ on chromosome 4 of 18.4 cM between markers D4S2623 and D4S1575 (Figure 1B). A search for candidate genes within this region revealed the $E G F$ gene, which was considered a highly relevant positional candidate since it had previously been linked to electrolyte homeostasis (16).

We sequenced EGF in the affected individuals (Figure 1A) and identified the homozygous mutation C3209T in exon 22, which cosegregated with the disorder and was absent in 126 ethnically matched control chromosomes (Figure 1C). The mutation caused the substitution of the highly conserved proline 1070 within the cytoplasmic tail of pro-EGF by leucine (P1070L) (Figure 1, D and E). We found that both parents and the 2 unaffected sisters (Figure 1A) of this Dutch family were heterozygous for the mutation. Furthermore, the paternal aunt was heterozygous for the mutation whereas her 2 children (Figure 1A) exhibited the homozygous wild-type genotype (Figure 1, A and C).

The EGF gene consists of 24 exons encoding a large type I membrane-anchored precursor protein of 1,207 amino acid residues that exists as a membrane-bound molecule, which is proteolytically cleaved into pro-EGF to finally generate the 53-amino acid peptide hormone EGF (17). EGF has a profound effect on cell differentiation in vivo and is a potent mitogenic factor for a variety of cultured cells of both ectodermal and mesodermal origin (18). EGF belongs to the EGF-like family of growth factors, which bind with high affinity to the EGFR. Other members of this family are TGF- $\alpha$, amphiregulin, heparin-binding EGF-like growth factor, betacellulin, and epiregulin (19-21). These membrane-anchored growth factor precursors are characterized by the fact that they are biologically active at the cell surface although they can be proteolytically cleaved to release soluble, diffusible factors (19-21).
To study the tissue distribution of pro-EGF and EGFR, we performed RT-PCR on various human tissues (Figure 2A). For EGF, PCR amplification products were detected in, e.g., kidney, salivary gland, cerebrum, and prostate, whereas no expression was detected in the adrenal gland, liver, cerebellum, and placenta. The EGFR showed a ubiquitous expression pattern since all tissues tested were positive by RT-PCR analysis (Figure 2A). Immunohistochemistry on rat kidney sections showed that EGF is consistently coexpressed with NCC, a marker of the DCT (22) (Figure 2B). Interestingly, the immunopositive staining is mainly localized along the apical domain in DCT. Coffey and coworkers demonstrated that human pro-EGF overexpressed in Madin-Darby canine kidney (MDCK) cells is delivered equally to the apical and basolateral membrane but is found predominantly at the apical membrane domain (23). They proposed that preferential ectodomain cleavage at the basolateral surface explains the apparent apical localization of pro-EGF. Sack and Talor provided evidence for the existence of specific binding sites in tubular basolateral membranes, suggesting a physiologic role of EGF in the kidney (24). In the kidney, the EGFR was detected in glomerular endothelial cells, peritubular capillaries, and arteriolar walls as well as along the thick ascending limb of Henle loop and DCT $(25,26)$. Immunohistochemical analysis revealed that the EGFR was located particularly along the basolateral membrane of the tubular cells. The DCT reabsorbs approximately $10 \%$ of the filtered $\mathrm{Mg}^{2+}$ load, and the reabsorption rate in this segment defines the final urinary $\mathrm{Mg}^{2+}$ excretion, as virtually no reabsorption takes place beyond this section (1). In kidney, the epithelial $\mathrm{Mg}^{2+}$ channel TRPM6 is specifically expressed in DCT, where it forms the rate-limiting step of epithelial $\mathrm{Mg}^{2+}$ transport (8). Thus, pro-EGF and TRPM6 are both predominantly 

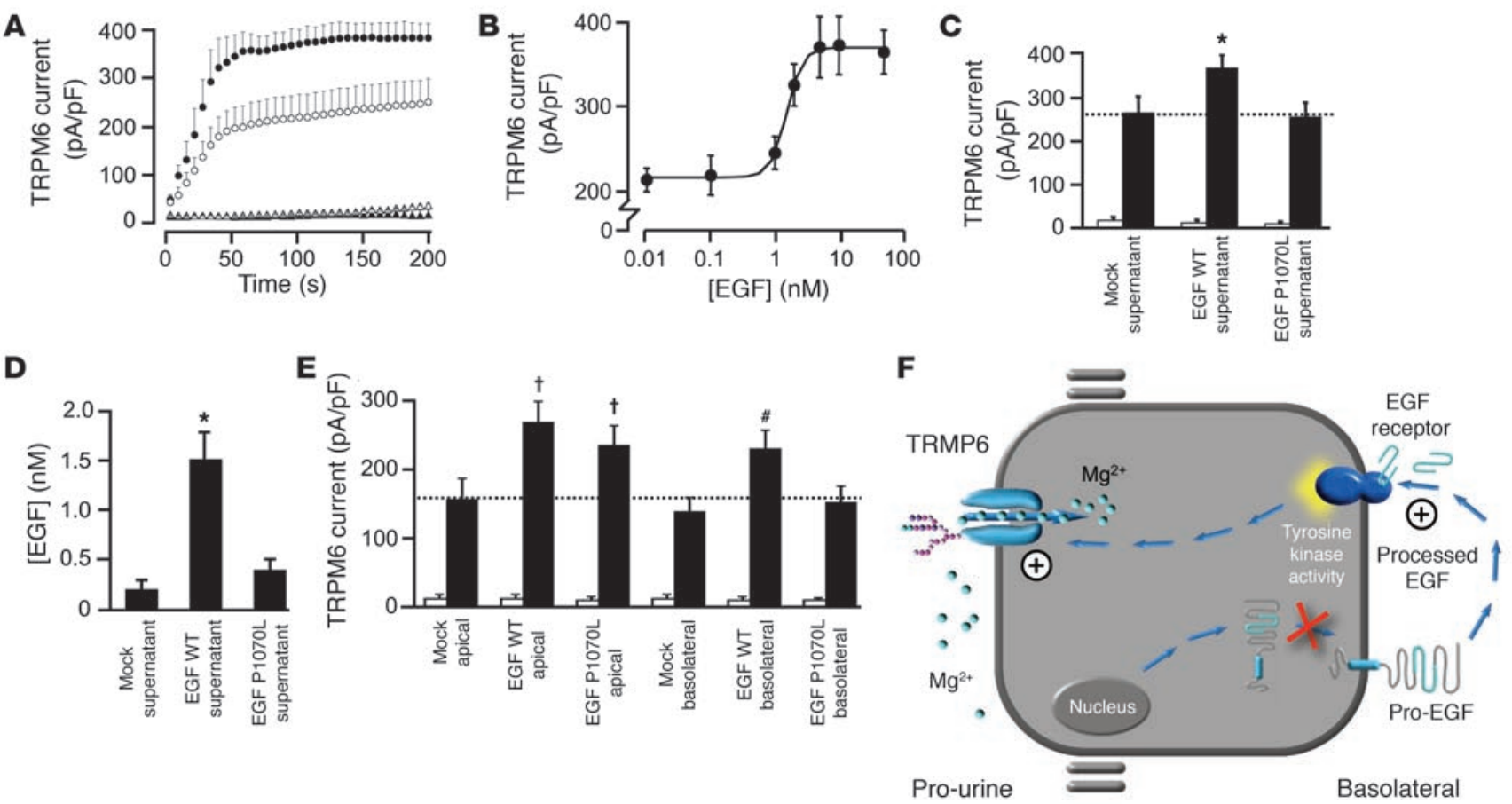

\section{Figure 3}

Electrophysiological analysis of pro-EGF and pro-EGF-P1070L on TRPM6 channel activity. (A) Average time course of outward (at +80 mV) current densities from HEK293 cells transfected with TRPM6 in control conditions (open circles) and after EGF treatment (30 minutes, 10 nM, filled circles) in comparison with mock-transfected cells without EGF treatment (triangles) and after EGF stimulation (filled triangles). $n=17-20$. (B) Dose-response curve of EGF-induced current in TRPM6-transfected HEK293 cells indicating half maximal effective concentration of $1.7 \mathrm{nM}$. $n=11-14$. (C) Histogram presenting the current densities at $+80 \mathrm{mV}$ of TRPM6-transfected cells (200 seconds after establishment of the wholecell configuration) treated with mock (mock), pro-EGF (EGF WT) or pro-EGF-P1070L (EGF-P1070L) supernatant. All treatments were performed for 30 minutes at $37^{\circ} \mathrm{C}$. White bars indicate experimental conditions in which HEK293 cells were transfected with mock DNA whereas black bars indicate HEK293 cells transfected with TRPM6. Asterisk indicates significance in comparison with nontreated TRPM6-transfected cells. $P=0.024 ; n=6$. (D) Supernatants of mock-, pro-EGF-, or pro-EGF-P1070L-transfected HEK293 cells were analyzed by ELISA. Asterisk indicates mock and pro-EGF-P1070L supernatant were significantly different from EGF supernatant. $P=0.006 ; n=4$. (E) Histogram summarizing the current density $(\mathrm{pA} / \mathrm{pF})$ at $+80 \mathrm{mV}$ (200 seconds after break-in) of HEK293 cells treated with the apical or basolateral supernatant of MDCK cells stably transfected with either wild-type pro-EGF, pro-EGF-P1070L, or mock DNA (30 minutes, $37^{\circ} \mathrm{C}$ ). White bars indicate experimental conditions in which HEK293 cells were transfected with mock DNA whereas black bars indicate HEK293 cells transfected with TRPM6. Crosses indicate significance in comparison with the mock treatment at the apical side (wild-type pro-EGF apical, $P=0.0001, n=6$; pro-EGF-P1070L apical, $P=0.029, n=6$ ), and pound symbol represents significance in comparison with the mock treatment at the basolateral side (wild-type pro-EGF basolateral $P=0.031, n=6$ ). (F) Schematic model illustrating how pro-EGF-P1070L mutation results in IRH. The pro-EGF-P1070L mutation leads to impaired basolateral sorting of pro-EGF, resulting in abrogated stimulation of the EGFR localized at the basolateral membrane. Activation of the EGFR in DCT by EGF is necessary to prevent renal $\mathrm{Mg}^{2+}$ wasting by stimulation of the epithelial Mg2+ channel TRPM6.

expressed in DCT, the main site of active renal $\mathrm{Mg}^{2+}$ reabsorption $(1,16,17,27)$. To determine whether EGF can regulate the activity of TRPM6, human embryonic kidney 293 (HEK293) cells were transiently transfected with TRPM6 and treated for 30 minutes with EGF (10 nM), resulting in increased channel activity (Figure 3A). Western blot analysis demonstrated endogenous expression of EGFR in these HEK293 cells (data not shown). EGF dose dependently stimulated TRPM6 activity with half maximal effective concentration of $1.7 \mathrm{nM}$, an apparent affinity that is in the physiological range as reported previously (Figure 3B) (24).

Previous in vivo studies showed that the predominant form of EGF released from epithelial cells is the high-molecular mass 160-170 kDa EGF, as found at high concentrations in serum, urine, and milk $(28,29)$. To investigate the functional effect of the identified mutation, HEK293 cells were transfected with wildtype or mutant pro-EGF and after 2 days their supernatant was collected. TRPM6-expressing HEK293 cells were subsequently incubated for 30 minutes with these preconditioned media and analyzed for channel activity using the patch clamp technique. Interestingly, the supernatant obtained from mutant pro-EGFexpressing HEK293 cells failed to stimulate TRPM6 activity whereas a significant channel activation was observed with the wild-type EGF-containing supernatant (Figure 3C). Quantification of EGF in both supernatants using ELISA indicated that the secretion of mutant pro-EGF is impaired (Figure 3D).

To demonstrate that the observed stimulatory effects are due to EGFR activation, a preincubation with cetuximab was performed. This IgG human/mouse chimeric monoclonal antibody competitively inhibits the activation of the EGFR. It binds externally to the EGFR to block binding of the ligand and subsequent signal transduction mediated via the receptor-associated tyrosine kinase and prevents phosphorylation of the EGFR and other downstream 
A

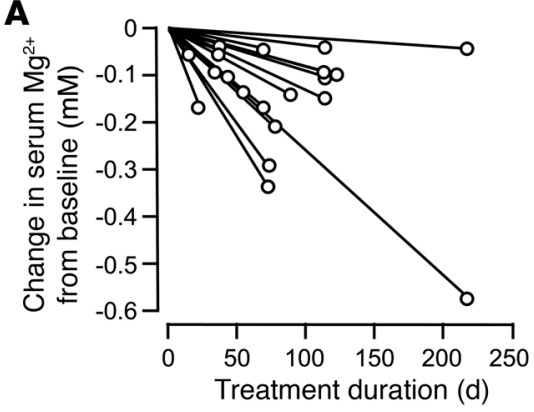

C

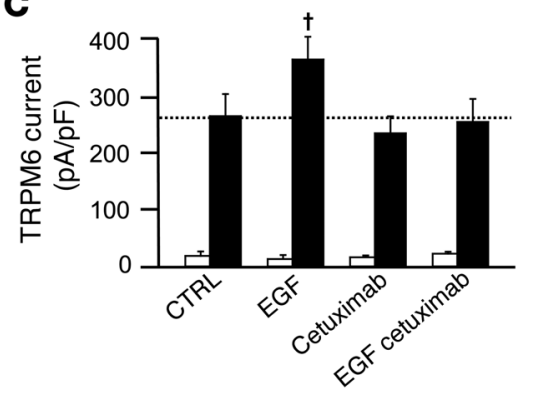

Figure 4

Effect of cetuximab treatment on $\mathrm{Mg}^{2+}$ balance and TRPM6 activity. (A) Changes in serum $\mathrm{Mg}^{2+}$ levels from baseline over time during cetuximab therapy are shown for 20 colorectal cancer patients. Solid lines represent individual linear regression lines of the data points for each individual patient. Open symbols denote end of treatment. (B) Cetuximab treatment leads to renal $\mathrm{Mg}^{2+}$ loss and hypomagnesemia. Serum samples and urine (over a 24-hour period) was collected from 8 patients at baseline in normomagnesemic conditions (open circles), 12 patients on cetuximab treatment in hypomagnesemic conditions (filled circles), and patients (V3, V4) with $\mathrm{IRH}$ (triangles), then analyzed for $\mathrm{FE}$ of $\mathrm{Mg}^{2+}$. $\mathrm{FE} \mathrm{Mg}^{2+}$ was plotted against the serum $\mathrm{Mg}^{2+}$ concentrations for the tested individuals. Hypomagnesemia in combination with an inappropriately high excretion of $\mathrm{Mg}^{2+}$ was observed in patients with the $\mathrm{P} 1070 \mathrm{~L}$ mutation as well as in individuals treated with cetuximab. Large circles represent the averaged values of patients on cetuximab treatment (filled) and patients at baseline in normomagnesemic conditions (open). Asterisk indicates a significant difference in serum $\left(\mathrm{Mg}^{2+}\right)$ compared with that in control patients. $P=0.001 ; n=8-12$. (C) Histogram depicting the current densities at $+80 \mathrm{mV}$ of TRPM6-transfected cells that were exposed for 30 minutes to EGF, cetuximab, or EGF in combination with cetuximab. Cross indicates a significant difference compared with nontreated (CTRL) HEK293TRPM6 cells. $P<0.026 ; n=6$.

proteins in the signal transduction cascade (30). The stimulatory effect of the supernatant collected from pro-EGF-expressing HEK293 cells on TRPM6 was significantly inhibited by preincubation for 1 hour with cetuximab $(191 \pm 22 \mathrm{pA} / \mathrm{pF}, 491 \pm 62 \mathrm{pA} / \mathrm{pF}$, and $286 \pm 48 \mathrm{pA} / \mathrm{pF}$ for TRPM6-expressing HEK293 cells measured at $+80 \mathrm{mV}$ and treated with mock supernatant, EGF supernatant, or EGF supernatant plus cetuximab, respectively; $P<0.05$, $n=6$ ). Thus, the stimulatory effect of the supernatant on TRPM6 activity is due to direct activation of the EGFR.

As outlined above, the localization of EGFR is restricted to the basolateral membrane whereas pro-EGF is present at both the apical and basolateral membranes (23). This prompted us to investigate in detail the cellular metabolism of pro-EGF. Recently, several basolateral sorting motifs were identified, including the sequence motif PXXP (with X being an arbitrary amino acid), determining the targeting of proteins to the basolateral membrane in MDCK cells (31). Our mutation substitutes the second highly conserved proline in the cytoplasmic 1067PKN 1070 motif into a leucine
(Figure 1D). We hypothesized that this mutation results in improper trafficking of pro-EGF, which prevents an adequate secretion of the hormone into the circulation. Since EGFR is only localized basolaterally, the mutation should prevent the ligand from reaching its receptor. Interestingly, $\mathrm{He}$ and coworkers have shown previously that a 22 -amino acid sequence in the EGFR juxtamembrane domain contains autonomous sorting information necessary for basolateral localization of the receptor in MDCK cells (31). This sorting motif contains a polyproline core comprising residues P667 and P670 (667PXXP670). Site-directed mutations at P667 and P670 were associated with impaired basolateral delivery of the EGFR.

To determine the effect of the mutation on the sorting of pro-EGF in polarized epithelial cells, MDCK cells stably expressing either wild-type or mutant proEGF were grown on semipermeable filter supports until they reached confluency. After 2 days, media of the basolateral and apical compartment were collected individually. Next, HEK293 cells expressing TRPM6 were treated with the collected preconditioned media of wild-type pro-EGF, mutant pro-EGF, or mockexpressing cells. Equal stimulation of TRPM6 channel activity was observed when the cells were incubated with the apically collected media of wild-type or mutant pro-EGF-expressing MDCK cells (Figure 3E). However, stimulation of TRPM6 channel activity was only observed with basolaterally collected wild-type pro-EGF medium, not with the basolateral mutant pro-EGF media (Figure 3E). Of note, the apically and basolaterally collected mock medium did not affect TRPM6 activity. These observations suggest that the basolateral release of mutant pro-EGF is diminished, seriously hampering the EGF-dependent activation of the basolaterally localized EGFR (Figure 3F).

Clinical trials directed to the treatment of patients with colorectal cancer have demonstrated that cetuximab is synergistic with chemotherapy for these patients (32). We observed that serum $\mathrm{Mg}^{2+}$ levels gradually decreased in all studied colorectal cancer patients on cetuximab treatment (Figure 4A). This is in line with a recent report showing that a significant number of similarly treated patients developed hypomagnesemia (30). Our patients treated with cetuximab displayed an $\mathrm{FE}$ of $\mathrm{Mg}^{2+}$ of 5.1\%, which is inappropriately high given their hypomagnesemia (Figure 4B). As previously established by Agus (5), an $\mathrm{FE}$ of $\mathrm{Mg}^{2+}$ above $2 \%$ in the presence of hypomagnesemia indicates renal $\mathrm{Mg}^{2+}$ loss.

The 2 affected sisters with IRH exhibited a similar relatively high $\mathrm{Mg}^{2+}$ excretion profile (Figure 4B). The urinary $\mathrm{Mg}^{2+}$ patterns of the patients treated with EGFR antibodies, the patients described in our Dutch family, and patients with hypomagnesemia with secondary hypocalcemia (HSH; OMIM 602014) $(11,12)$ were identical and suggest mutual defects in renal TRPM6 activity. Indeed, preincubation with cetuximab abolished the stimulatory effect of EGF on TRPM6 activity as shown by patch clamp analysis of TRPM6-expressing HEK293 cells (Figure 4C). Taken together, these data strongly suggest a pivotal role for EGFR signaling in the maintenance of normal $\mathrm{Mg}^{2+}$ balance and indicate TRPM6 as a critical link between EGFR inhibition and IRH. 
Through the discovery of an EGF mutation in IRH, we have for the first time, to our knowledge, identified a magnesiotropic hormone crucial for total body $\mathrm{Mg}^{2+}$ balance that directly stimulates renal tubular $\mathrm{Mg}^{2+}$ reabsorption via activation of the epithelial $\mathrm{Mg}^{2+}$ channel TRPM6. Moreover, it raises the question of whether EGF plays a role in mental development since our IRH patients (Figure 1A) are mentally retarded. EGF is present in cerebrospinal fluid, and EGF mRNA has been detected in the developing brain of various species. Furthermore, EGF is a well-known neurotrophic factor regulating the development of various neuronal cells (33). Futamura et al. reported that serum EGF levels were markedly decreased in schizophrenic patients and suggested that EGF might provide a link between impaired EGF signaling and the pathology/etiology of schizophrenia (34). An association between an EGF polymorphism and schizophrenia has been suggested although data from several studies are conflicting $(35,36)$. Interestingly, in our Dutch family with IRH, 1 unaffected, EGF P1070L-carrier sister (Figure 1A) suffers from schizophrenia, and 2 brothers and 1 sister of the carrier mother (Figure $1 \mathrm{~A}$ ) are known to have cluster A (Diagnostic and statistical manual of mental disorders, fourth edition, text revision) personality disorders. In addition, EGF could be involved in $\mathrm{Mg}$ absorption in the intestine, a site that also expresses TRPM6. If not, please revise sentence. Future therapeutic intervention might be able to treat hypo- and hypermagnesemia by regulating EGFR activity.

\section{Methods}

Statistics. In all experiments, data are expressed as mean \pm SEM. Overall statistical significance was determined by ANOVA. Where differences between the means of 2 groups were significant, they were analyzed by pairwise comparison using Scheffe's method. For the statistical analysis in Figure 4B, an unpaired Student's $t$ test was performed. $P<0.05$ was considered significant. Statistical analysis was performed using SPSS software 12.0 (SPSS).

Subjects. Informed consent was obtained from the parents and unaffected daughters of the family in this study. The parents gave proxy consent for the 2 affected daughters in this study. In this family, 2 Dutch sisters suffered from primary renal $\mathrm{Mg}^{2+}$ loss associated with normocalciuria. These women are the offspring of a consanguineous mating, and since both parents did not display this disorder, the inheritance pattern was likely to be autosomal recessive. The clinical data of this family have previously been described in detail by Geven et al. (14). In short, 2 patients of this family, $\mathrm{V} 3$ and $\mathrm{V} 4$, had serum $\mathrm{Mg}^{2+}$ levels of $0.53 \mathrm{mM}$ and $0.56 \mathrm{mM}$ and urinary $\mathrm{Mg}^{2+}$ values of $3.9 \mathrm{mmol} / 24 \mathrm{~h}$ and $3.7 \mathrm{mmol} / 24 \mathrm{~h}$, respectively. Given the low serum $\mathrm{Mg}^{2+}$ levels, urinary $\mathrm{Mg}^{2+}$ excretion was relatively high, indicating a renal $\mathrm{Mg}^{2+}$ reabsorption defect. Both patients suffer from epileptic seizures that started in their first year of life and are controlled by conventional antiepileptic drugs. During childhood, they showed psychomotor retardation, and they are presently moderately mentally retarded women. Serum and urinary $\mathrm{Ca}^{2+}$ were in the normal range, as were serum $\mathrm{Na}^{+}, \mathrm{K}^{+}$, $\mathrm{Cl}^{-}, \mathrm{HCO}_{3}{ }^{-}$, and blood $\mathrm{pH}$ values. In both patients, plasma renin activity, plasma aldosterone, and parathyroid hormone concentrations were in the normal range. Blood was collected for genotyping from the parents, both affected and both unaffected daughters, the sister of the father, and the sister's 2 unaffected children.

Mutation analysis. We extracted DNA using standard protocols. The exons of the $E G F$ gene were amplified separately from genomic DNA, using the primers listed in Supplemental Table 1 (supplemental material available online with this article; doi:10.1172/JCI31680DS1). PCR products were purified with QIAGEN PCR Purification Kit according to the manufacturer's protocol, and products were sequenced using the same primers.
Mutation analysis of the EGF gene was performed by forward sequencing of the coding regions of the gene of the youngest affected family member. Forward and reverse sequencing of exon 22 was subsequently performed on all family members.

Subjects with colorectal cancer treatment. We prospectively studied consecutive patients with metastatic colorectal cancer for which treatment with cetuximab was initiated at the University Hospital Gasthuisberg (Leuven, Belgium). Most patients were included in phase II or III trials. Cetuximab was kindly donated by Merck KGaA. Informed consent was obtained from all subjects investigated in this study.

Calculation of $F E_{M g}$. The $F E_{M g}$ was calculated using the following formula:

\section{Equation 1}

$$
F E_{M g}=\frac{U_{M g} \times P_{C r}}{\left(0.7 \times P_{M g}\right) \times U_{G r}} \times 100
$$

$U$ and $P$ refer to the urine and serum concentrations of $\mathrm{Mg}^{2+}(\mathrm{Mg})$ and creatinine $(\mathrm{Cr})$. Serum $\mathrm{Mg}^{2+}$ concentration was multiplied by 0.7 since only approximately $70 \%$ of the circulating $\mathrm{Mg}^{2+}$ was unbound by albumin and therefore able to be filtered across the glomerulus.

Expression profiling. PCRs were performed in $50 \mu \mathrm{l}$ reaction volumes containing $1 \mu 1$ of cDNA, $10 \mathrm{pmol}$ of each primer (Supplemental Table 2), $2.5 \mathrm{mM} \mathrm{MgCl}_{2}, 200 \mu \mathrm{M}$ of each deoxyribonucleotide triphosphate (dATP, dTTP, dCTP, and dGTP), and $0.5 \mathrm{U}$ of Taq polymerase. PCRs had an initial denaturation stage of 1 minute at $95^{\circ} \mathrm{C}$, followed by 39 cycles of $30 \mathrm{sec}-$ onds at $95^{\circ} \mathrm{C}, 1$ minute at $55^{\circ} \mathrm{C}, 1$ minute at $72^{\circ} \mathrm{C}$, and a final extension step at $72^{\circ} \mathrm{C}$ for 10 minutes.

Immunohistochemistry. Staining of rat kidney sections for NCC and EGF was performed on $7-\mu \mathrm{m}$ cryosections of periodate-lysine paraformaldehydefixed kidney samples. Sections were stained with affinity-purified anti-NCC (1:200) and affinity-purified rabbit anti-EGF (1:100) (Calbiochem; EMD Biosciences). Images were made with a Bio-Rad MRC 1000 laser scanning confocal imaging system using a $\times 60$ oil-immersion objective.

Linkage analysis. Samples of peripheral EDTA blood were collected from each person, and genomic DNA was isolated using standard procedures. Homozygosity mapping was performed with a genome-wide set of 400 evenly distributed microsatellite markers (LMS-MD10 2.5; Applied Biosystems), with an average intermarker distance of $10 \mathrm{cM}$, on the basis of Marshfield genetic maps (http://research.marshfieldclinic.org/genetics/ GeneticResearch/compMaps.asp). The average heterozygosity of these markers was 0.76. Five additional markers, D4S1570, D4S2623, D4S2392, D4S1615, and D4S422, were selected for fine mapping purposes from the public databases on chromosome 4 (http://research.marshfieldclinic.org/ genetics/GeneticResearch/data/Maps/Map4.txt and http://genome.ucsc. edu/cgi-bin/hgGateway. The analysis of the markers was according to the protocol provided for the LMS-MD10 version 2.5 with some small modifications. PCRs were performed in $10 \mu \mathrm{l}$ reaction volumes containing $40 \mathrm{ng}$ of genomic DNA, 5 pmol of each primer, $2.5 \mathrm{mM} \mathrm{MgCl}_{2}, 250 \mu \mathrm{M}$ dATP, dTTP, dCTP, and dGTP each, $10 \mathrm{mM}$ Tris/HCl (pH 8.3), $50 \mathrm{mM} \mathrm{KCl}$, and $0.4 \mathrm{U}$ of Taq polymerase (AmpliTaq TM Gold; Applied Biosystems). PCRs had an initial denaturation stage of 12 minutes at $95^{\circ} \mathrm{C}$, followed by 10 cycles of 15 seconds at $94^{\circ} \mathrm{C}, 15$ seconds at $55^{\circ} \mathrm{C}$, and 30 seconds at $72^{\circ} \mathrm{C}$, then 22 cycles of 15 seconds at $89^{\circ} \mathrm{C}, 15$ seconds at $55^{\circ} \mathrm{C}$, and 30 seconds at $72^{\circ} \mathrm{C}$, and a final extension step at $72^{\circ} \mathrm{C}$ for 10 minutes. Reactions for each marker were performed separately, with products being pooled into size-specific sets before typing. Markers were typed on an ABI 3100 sequencer (Applied Biosystems) using GeneMapper 4.0 software (Applied Biosystems). Allele binning was performed with the Excel 2000 (Microsoft) macro linkage designer developed by van Camp and coworkers (37), and 
we checked Mendelian inheritance of alleles with PedCheck 1.0 software (http://watson.hgen.pitt.edu) (38). Multipoint LOD scores were calculated with the GeneHunter, version 2.1 release 5, program in the easyLINKAGE software package (39).

Constructs. The full-length EGF precursor in vector plasmid SAD (pSAD) was kindly provided by G.I. Bell (17) (University of Chicago, Chicago, Illinois, USA). The full-length EGF precursor P1070L mutant was obtained by in vitro mutagenesis of the PSAD-EGF vector according to the protocol described by the manufacturer (Invitrogen). The construct was verified by DNA sequence analysis. To generate plasmid CB7 constructs for the stable expression of the full-length EGF precursor and the P1070L mutant in MDCK type I cells, PCR was performed on the PSAD-EGF and PSAD-EGFP1070L constructs, respectively, using Pyrococcus furiosus DNA polymerase according to the manufacturer's protocol (Stratagene). Primers used were 5'-CGGGGTACCGCCACCATGCTGCTCACTCTTATCATTC-3' and 5'-CGCTCTAGAGTCACTGAGTCAGCTCCATTTG-3'. PCR products were cloned into the plasmid CB7 vector using the restriction enzymes $\mathrm{KpnI}$ and $\mathrm{XbaI}$ and verified by DNA sequence analysis.

Electrophysiology. The bicistronic expression vector pCINeo-IRES-GFP containing the full-length open reading frames of $\mathrm{N}$ terminally HAtagged human TRPM6 (GenBank accession number NM_017662) was used to coexpress human TRPM6 and enhanced GFP in HEK293 cells. HEK293 cells were grown in DMEM (BioWhittaker) containing 10\% (v/v) fetal calf serum, $2 \mathrm{mM}$ L-glutamine, and $10 \mu \mathrm{g} / \mathrm{ml}$ Ciproxin at $37^{\circ} \mathrm{C}$ in a humidity-controlled incubator with $5 \%(\mathrm{v} / \mathrm{v}) \mathrm{CO}_{2}$. Cells were transiently transfected with the respective constructs using Lipofectamine 2000 (Invitrogen), as described previously (40), and electrophysiological recordings were performed 48 hours after transfection. Transfected cells were identified by their green fluorescence when illuminated at $480 \mathrm{~nm}$. Nontransfected (GFP-negative) cells from the same batch were used as controls. Patch clamp experiments were performed in the tight seal wholecell configuration at room temperature $\left(20-25^{\circ} \mathrm{C}\right)$ using an EPC-10 patch clamp amplifier computer controlled by PatchMaster Classic 1.20 software (HEKA Elektronik). Currents were digitized at $10 \mathrm{kHz}$ and digitally filtered at $2.9 \mathrm{kHz}$. Patch pipettes had resistances between 2 and $5 \mathrm{MW}$ after filling with the standard intracellular solution. Cells were held at $0 \mathrm{mV}$, and voltage ramps of $200 \mathrm{~ms}$ duration ranging from -100 to +100 $\mathrm{mV}$ were applied every 2 seconds. Extracting the current amplitudes at $+80 \mathrm{mV}$ from individual ramp current records provided an assessment of the temporal development of membrane currents. Current densities were obtained by normalizing the current amplitude to the cell membrane capacitance. The time course of current development was determined by measuring the current at $+80 \mathrm{mV}$. Step protocols were applied from holding potentials of $0 \mathrm{mV}$ and consisted of 400 -ms steps to -100 to $+100 \mathrm{mV}$ (increment of $20 \mathrm{mV}$ ). The standard pipette solution contained $150 \mathrm{mM}$ $\mathrm{NaCl}, 10 \mathrm{mM}$ EDTA, and $10 \mathrm{mM}$ HEPES/NaOH (pH 7.2). Extracellular solutions contained $150 \mathrm{mM} \mathrm{NaCl}$ and $10 \mathrm{mM}$ HEPES/NaOH (pH 7.4) supplemented with $1 \mathrm{mM} \mathrm{CaCl}_{2}$. The osmolarity of the solutions was adjusted to $300-310 \mathrm{mOsm} / \mathrm{kg}$ with mannitol.

Pretreatment with EGF, preconditioned media, and cetuximab. The effect of EGF on TRPM6 activity in HEK293 cells transiently transfected with TRPM6 was measured after a 30-minute preincubation with $10 \mathrm{nM}$ mouse EGF (Sigma-Aldrich) in DMEM (modified as described above) or the preconditioned medium. Cetuximab pretreatment for 1 hour with $2 \mu \mathrm{g} / \mathrm{ml}$ in DMEM (modified as described above) was performed prior to treatment with EGF or preconditioned medium. Subsequently, cells were analyzed by the patch clamp technique as described above.

Generation of an MDCK cell line stably expressing pro-EGF and pro-EGFP1070L. MDCK type I cells (41) were grown in DMEM containing 5\% (v/v) FCS (HyClone), $13 \mathrm{mM} \mathrm{NaHCO}_{3}, 2 \mathrm{mM} \mathrm{L-glutamine,} \mathrm{and} 10 \mu \mathrm{g} / \mathrm{ml}$ ciproxin (Bayer) at $37^{\circ} \mathrm{C}$ in a humidity-controlled incubator with $5 \%$ $(\mathrm{v} / \mathrm{v}) \mathrm{CO}_{2}$. For stable transfection of MDCK cells, $25 \mu \mathrm{g}$ circular DNA was transfected using the $\mathrm{Ca}^{2+}$-phosphate precipitation technique (42). Cells were trypsinized 24 hours after transfection, seeded on Petri dishes, and cultured in DMEM containing $75 \mu \mathrm{g} / \mathrm{ml}$ hygromycin B (Invitrogen). Between 10 and 14 days after transfection, individual colonies were selected by means of cloning rings and expanded. Total RNA was extracted from each colony that had been grown until confluent in a 24-well culture plate using TRIzol Total RNA Isolation Reagent (Invitrogen) according to the manufacturer's protocol. The obtained RNA was subjected to DNase treatment (Promega) to prevent genomic DNA contamination. Thereafter, $1.5 \mu \mathrm{g}$ of RNA was reverse transcribed by Moloney Murine Leukemia Virus Reverse Transcriptase (Invitrogen) as described previously (43). The cDNA was used to determine pro-EGF expression with the primers described in Supplemental Table 2. MDCK cells stably expressing plasmid CB7-EGF, plasmid CB7-EGF-P1070L, or empty vector (5 clones of each) were used to seed $1024-\mathrm{mm}$ Transwell filter wells of each condition (Corning Inc.). We seeded $1.5 \times 10^{5} \mathrm{cells} / \mathrm{cm}^{2}$ on permeable filter supports; these were grown for 4 days until they reached confluency and an electrical resistance of greater than $2,400 \Omega \cdot \mathrm{cm}^{2}$. Next, the media were replaced by DMEM medium as described below but without FCS and harvested after 2 days.

Transfection of HEK293 cells. HEK293 cells were transiently transfected with pSAD-EGF, pSAD-EGF-P1070L, or empty-vector pCINeo-IRES-GFP cDNA using polyethylenimine (Polysciences Inc.). Six million cells were seeded on a petri dish $\left(57 \mathrm{~cm}^{2}\right)$ (10 plates per vector) in a total volume of $5 \mathrm{ml}$ DMEM. After 3 hours, the cells were transfected. The transfection mix for each plate consisted of $15 \mu \mathrm{g}$ cDNA and $90 \mu \mathrm{l}$ polyethylenimine $(1 \mu \mathrm{g} / \mu \mathrm{l})$ in a total volume of $500 \mu \mathrm{l}$ Opti-MEM (Invitrogen). The transfection mix was incubated for 20 minutes at room temperature. Subsequently, the transfection mix was added to the cells and incubated overnight. After 1 day of transfection, the culture media was replaced by $8 \mathrm{ml}$ DMEM medium as described above but without FCS.

Collection and concentration of conditioned culture media. Two days after replacement of the medium of HEK293 and MDCK cells, the FCS-free culture media was collected and proteinase inhibitors were added (1 mM PMSF, $1 \mu \mathrm{g} / \mathrm{ml}$ pepstatin A, and $10 \mathrm{ng} / \mathrm{ml}$ leupeptin). Subsequently, the collected FCS-free culture media of HEK293 cells with added proteinase inhibitors was concentrated 400 times using Centriprep YM-3 followed by Microcon YM-3 (Millipore). Prior to concentration of the collected HEK293 medium, $2 \mathrm{ml}$ of the collected FCS-free culture medium was kept separately and used for patch clamp analysis.

Human EGF ELISA. EGF concentrations were determined in 400 times concentrated culture supernatant by using Centriprep columns and a human EGF ELISA kit according the manufacturer's protocol (RayBiotech Inc.).

\section{Acknowledgments}

The authors thank H. van Bokhoven, F. Sweep, and G. Bell for providing genomic control DNA, blood samples, and the cDNA encoding human pro-EGF, respectively. We acknowledge J. van Zoelen for generously donating the antibody against pro-EGF and helpful discussions. The authors are grateful to A. van der Kemp for immunohistochemical analysis, K. Lee for electrophysiological measurements, and A. Heister for linkage studies. This study was supported financially by the Dutch Kidney Foundation (C02.2030 and C03.6017). The authors were further supported by grants from the European Science Foundation (EURYI 2006), the Netherlands Organization for Scientific Research (ZonMW 9120.6110), and the Human Frontier Science Program (RGP32/2004). S. Thébault is supported by an EMBO long-term fellowship (ALTF 727-2005), 
and S. Tejpar and E. van Cutsem are senior clinical investigators of the Fund for Scientific Research - Flanders (FWO).

Received for publication January 31, 2007, and accepted in revised form May 8, 2007.

1. Dai, L.J., et al. 2001. Magnesium transport in the renal distal convoluted tubule. Physiol. Rev. 81:51-84.

2. Ferment, O., Garnier, P.E., and Touitou, Y. 1987. Comparison of the feedback effect of magnesium and calcium on parathyroid hormone secretion in man. J. Endocrinol. 113:117-122.

3. Cholst, I.N., et al. 1984. The influence of hypermagnesemia on serum calcium and parathyroid hormone levels in human subjects. N. Engl. J. Med. 310:1221-1225.

4. Konrad, M., Schlingmann, K.P., and Gudermann, T. 2004. Insights into the molecular nature of magnesium homeostasis. Am. J. Physiol. Renal. Physiol. 286:F599-F605.

5. Agus, Z.S. 1999. Hypomagnesemia. J. Am. Soc. Nephrol. 10:1616-1622.

6. Quamme, G.A. 1997. Renal magnesium handling: new insights in understanding old problems. Kidney Int. 52:1180-1195.

7. Hoenderop, J.G., and Bindels, R.J. 2005. Epithelial $\mathrm{Ca}^{2+}$ and $\mathrm{Mg}^{2+}$ channels in health and disease. J. Am. Soc. Nephrol. 16:15-26.

8. Voets, T., et al. 2004. TRPM6 forms the $\mathrm{Mg}^{2+}$ influx channel involved in intestinal and renal $\mathrm{Mg}^{2+}$ absorption. J. Biol. Chem. 279:19-25.

9. Simon, D.B., et al. 1996. Genetic heterogeneity of Bartter's syndrome revealed by mutations in the $\mathrm{K}^{+}$ channel, ROMK. Nat. Genet. 14:152-156.

10. Meij, I.C., et al. 2003. Dominant isolated renal magnesium loss is caused by misrouting of the $\mathrm{Na}^{+}, \mathrm{K}^{+}$-ATPase gamma-subunit. Ann. N. Y. Acad. Sci. 986:437-443.

11. Schlingmann, K.P., et al. 2002. Hypomagnesemia with secondary hypocalcemia is caused by mutations in TRPM6, a new member of the TRPM gene family. Nat. Genet. 31:166-170.

12. Walder, R.Y., et al. 2002. Mutation of TRPM6 causes familial hypomagnesemia with secondary hypocalcemia. Nat. Genet. 31:171-174.

13. Sutton, R.A., and Domrongkitchaiporn, S. 1993. Abnormal renal magnesium handling. Miner. Electrolyte Metab. 19:232-240.

14. Geven, W.B., Monnens, L.A., Willems, J.L., Buijs, W., and Hamel, C.J. 1987. Isolated autosomal recessive renal magnesium loss in two sisters. Clin. Genet. 32:398-402.

15. Meij, I.C., et al. 2003. Exclusion of mutations in FXYD2, CLDN16 and SLC12A3 in two families with primary renal $\mathrm{Mg}^{2+}$ loss. Nephrol. Dial. Transplant. 18:512-516.

16. Rall, L.B., et al. 1985. Mouse prepro-epidermal growth factor synthesis by the kidney and other nology. 132:417-425.

Address correspondence to: René J. Bindels, 286 Cell Physiology, Nijmegen Centre for Molecular Life Sciences, Radboud University Nijmegen Medical Centre, PO Box 9101, 6500 HB Nijmegen, The Netherlands. Phone: 31-24-3614211; Fax: 31-24-3616413; E-mail: r.bindels@ncmls.ru.nl.

tissues. Nature. 313:228-231.

17. Bell, G.I., et al. 1986. Human epidermal growth factor precursor: cDNA sequence, expression in vitro and gene organization. Nucleic Acids Res. 14:8427-8446.

18. Gray, A., Dull, T.J., and Ullrich, A. 1983. Nucleotide sequence of epidermal growth factor cDNA predicts a 128,000 -molecular weight protein precursor. Nature. 303:722-725.

19. Barnard, J.A., Beauchamp, R.D., Russell, W.E., Dubois, R.N., and Coffey, R.J. 1995. Epidermal growth factor-related peptides and their relevance to gastrointestinal pathophysiology. Gastroenterology. 108:564-580.

20. Derynck, R. 1992. The physiology of transforming growth factor-alpha. Adv. Cancer Res. 58:27-52.

21. Lee, D.C., Fenton, S.E., Berkowitz, E.A., and Hissong, M.A. 1995. Transforming growth factor alpha: expression, regulation, and biological activities. Pharmacol. Rev. 47:51-85.

22. Loffing, J., et al. 2001. Distribution of transcellular calcium and sodium transport pathways along mouse distal nephron. Am. J. Physiol. Renal Physiol. 281:F1021-F1027.

23. Dempsey, P.J., Meise, K.S., Yoshitake, Y., Nishikawa, K., and Coffey, R.J. 1997. Apical enrichment of human EGF precursor in Madin-Darby canine kidney cells involves preferential basolateral ectodomain cleavage sensitive to a metalloprotease inhibitor. J. Cell Biol. 138:747-758.

24. Sack, E., and Talor, Z. 1988. High affinity binding sites for epidermal growth factor (EGF) in renal membranes. Biochem. Biophys. Res. Commun. 154:312-317.

25. Salido, E.C., Barajas, L., Lechago, J., Laborde, N.P., and Fisher, D.A. 1986. Immunocytochemical localization of epidermal growth factor in mouse kidney. J. Histochem. Cytochem. 34:1155-1160.

26. Gesualdo, L., et al. 1996. Expression of epidermal growth factor and its receptor in normal and diseased human kidney: an immunohistochemical and in situ hybridization study. Kidney Int. 49:656-665.

27. Salido, E.C., Fisher, D.A., and Barajas, L. 1986. Immunoelectron microscopy of epidermal growth factor in mouse kidney. J. Ultrastruct. Mol. Struct. Res. 96:105-113.

28. Lakshmanan, J., Salido, E.C., Lam, R., and Fisher, D.A. 1992. Epidermal growth factor prohormone is secreted in human urine. Am. J. Physiol. 263:E142-E150.

29. Mroczkowski, B., and Reich, M. 1993. Identification of biologically active epidermal growth factor precursor in human fluids and secretions. Endocri-

30. Schrag, D., Chung, K.Y., Flombaum, C., and Saltz, L.
2005. Cetuximab therapy and symptomatic hypomagnesemia. J. Natl. Cancer Inst. 97:1221-1224.

31. He, C., Hobert, M., Friend, L., and Carlin, C. 2002. The epidermal growth factor receptor juxtamembrane domain has multiple basolateral plasma membrane localization determinants, including a dominant signal with a polyproline core. J. Biol. Chem. 277:38284-38293.

32. Cunningham, D., et al. 2004. Cetuximab monotherapy and cetuximab plus irinotecan in irinotecan-refractory metastatic colorectal cancer. N. Engl. J. Med. 351:337-345.

33. Wong, R.W., and Guillaud, L. 2004. The role of epidermal growth factor and its receptors in mammalian CNS. Cytokine Growth Factor Rev. 15:147-156.

34. Futamura, T., et al. 2002. Abnormal expression of epidermal growth factor and its receptor in the forebrain and serum of schizophrenic patients. Mol. Psychiatry. 7:673-682.

35. Anttila, S., et al. 2004. Association of EGF polymorphism with schizophrenia in Finnish men. Neuroreport. 15:1215-1218.

36. Watanabe, Y., Fukui, N., Muratake, T., Kaneko, N., and Someya, T. 2005. No association of EGF polymorphism with schizophrenia in a Japanese population. Neuroreport. 16:403-405.

37. Van Camp, G., Balemans, W., and Willems, P.J. 1997. Linkage designer and linkage reporter software for automated gene localization studies. Technical Tips Online. 1:40013.

38. O'Connell, J.R., and Weeks, D.E. 1998. PedCheck: a program for identification of genotype incompatibilities in linkage analysis. Am. J. Hum. Genet. 63:259-266.

39. Collin, R.W., et al. 2007. Involvement of DFNB59 mutations in autosomal recessive nonsyndromic hearing impairment. Hum. Mutat. 28:718-723.

40. Chang, Q., et al. 2005. The beta-glucuronidase klotho hydrolyzes and activates the TRPV5 channel. Science. 310:490-493.

41. Richardson, J.C., Scalera, V., and Simmons, N.L. 1981. Identification of two strains of MDCK cells which resemble separate nephron tubule segments. Biochim. Biophys. Acta. 673:26-36.

42. Deen, P.M., Nielsen, S., Bindels, R.J., and van Os, C.H. 1997. Apical and basolateral expression of aquaporin-1 in transfected MDCK and LLC-PK cells and functional evaluation of their transcellular osmotic water permeabilities. Pflugers Arch. 433:780-787.

43. Hoenderop, J.G., et al. 2000. Localization of the epithelial $\mathrm{Ca}^{2+}$ channel in rabbit kidney and intestine. J. Am. Soc. Nephrol. 11:1171-1178. 